\title{
Improving end-of-life communication between primary and secondary care within respiratory medicine
}

\author{
To the Editor:
}

We read with interest the editorial about "Improving palliative care for patients with COPD" [1] and the subsequent article by MEFFET et al. [2]. We would like to highlight the results of our study looking at the impact of initiatives to improve communication with primary care in relation to end-of-life care (EoLC).

In the UK, recent controversies surrounding the Liverpool Care Pathway emphasised the importance of communication with regard to EoLC [3]. There are a number of local, regional and national strategies that are promoting EoLC. The Department of Health EoLC Strategy focuses on allowing patients to have a choice about end-of-life decisions, therefore facilitating a better quality of life when entering the terminal phase of their disease [4]. It highlights that good communication of information between fellow professionals as well as between professionals and their patients and families is essential. The key drivers to achieve this are the Electronic Palliative Care Co-ordination System, Advance Care Planning, AMBER care bundle [5], Rapid Discharge Pathway and Care of the Dying Patient. More recently, the document published by the Leadership Alliance, in response to the Neuberger report on the Liverpool Care Pathway, emphasised individualised care based on the patient's wishes. It also again highlights the importance of communication with the patient and their loved ones [6].

Locally, Leeds Teaching Hospitals National Health Service (NHS) Trust is one of the flagships in the NHS Transforming End of Life Care in Acute Hospitals project. A senior clinician development programme to improve EoLC within medical and surgical specialities was instigated by the Trust in 2011 for a 1-year period. This enabled the clinicians to take a lead on EoLC within their individual speciality and also promoted integration of EoLC across the primary/secondary care interface. A number of other educational initiatives were introduced during this time, including the AMBER care bundle which aims to improve the identification of clinically unwell inpatients, with limited reversibility and at risk of death within the next few months. This care bundle emphasised the need for discussion of issues including ceiling of care, resuscitation and preferred place of care, as appropriate for the patient. The ultimate aim being to improve communication and allow patients input into their care plans.

In our study we reviewed all deaths as an inpatient and within 28 days of discharge, from the respiratory wards, for the same 6-month periods during 2011 and 2013. The data were collected through the patient administration system database and electronic patient records. A retrospective review of discharge summaries and clinic letters only from the respiratory department was undertaken. Any mention of EoLC, Gold Standard Framework, palliative care, fast-track discharge or best supportive care was counted as an appropriate communication with primary care. Data relating to the cause of death were recorded.

Table 1 shows that over the study period there was a significant decrease in the proportion of inpatient deaths despite an increase in activity (Chi-squared, $\mathrm{p}<0.001$ ). There was also a corresponding increase in the proportion of deaths post-discharge. During this period there were also significant improvements in written communication with primary care of issues surrounding EoLC in the preceding year. This was for both patients dying post-discharge and as an inpatient. Those who died within 28 days of discharge were more likely to have a malignant diagnosis (see table 1) and the majority of these patients had documented communication of palliative intent. Over the study period there was a greater proportion of patients discharged from hospital with a nonmalignant diagnosis and communication with primary care (table 1) with regard to a palliative intent to therapy.

During the 2-year per study period the number of admissions increased, but there was a reduction in the proportion of patients dying in the Department of Respiratory Medicine. We cannot be sure that the improvements in communication with primary care were responsible for this as there were a number of improvements directed towards EoLC being undertaken. Communication is a critical step in organising high-quality EoLC for our patients and we need to be conscious that an acute admission to hospital is a significant event for patients with any respiratory disease, but particularly chronic obstructive pulmonary disease (COPD) in terms of prognostication [7]. For patients who died both as inpatients and within 28 days of discharge we only examined previous discharge summaries, clinic letters and any other communication from within the Department of Respiratory Medicine. We may therefore have 
TABLE 1 Breakdown of inpatient and outpatient deaths 2011-2013

\begin{tabular}{lccc} 
& $\mathbf{2 0 1 1}$ & $\mathbf{2 0 1 3}$ & p-value \\
\hline Admissions & 2148 & 2373 & \\
Inpatient deaths & $144(6.7)$ & $112(4.7)$ & $<0.001$ \\
EoLC mentioned in communication with primary care in preceding year & $78(54.2)$ & $75(67.0)$ & 0.002 \\
Cause of death & & & \\
$\quad$ Lung malignancy & $25(17.4)$ & $12(10.7)$ & $<0.001$ \\
$\quad$ Non-lung malignancy & $3(2.1)$ & $2(1.8)$ & 0.002 \\
$\quad$ Respiratory disease & $99(68.8)$ & $81(72.3)$ & $<0.001$ \\
Other & $17(11.8)$ & $17(15.2)$ & 0.002 \\
Post-discharge deaths & $37(1.9)$ & $48(2.1)$ & $<0.001$ \\
EoLC mentioned in communication with primary care & $31(83.8)$ & $47(97.2)$ & 0.005 \\
Diagnosis at discharge & & & \\
$\quad$ Lung malignancy & $22(59.5)$ & $19(39.6)$ & 0.084 \\
Non-lung malignancy & $2(5.4)$ & $4(8.3)$ & 0.061 \\
Respiratory disease & $7(18.9)$ & $20(41.7)$ & 0.001 \\
Other & $6(16.2)$ & $5(10.4)$ & 0.099 \\
\hline
\end{tabular}

Data are presented as $\mathrm{n}$ or $\mathrm{n}(\%)$, unless otherwise stated. EoLC: end-of-life care.

underestimated the proportion of patients who were labelled as requiring EoLC. These patients may have been seen by other specialities, including oncology and general medicine, that may have identified this group via communication with the general practitioner that we did not examine.

It is also important to remember that there will always be a proportion of unexpected deaths in the community and in hospital, and we cannot identify every patient. However, we can identify patients at risk of death, and it is essential to communicate this to the patient, family/carers and critically primary care to enable facilitation of high-quality EoLC. Communication with primary care regarding EoLC was more robust for patients with a malignant diagnosis compared with those with a nonmalignant diagnosis such as COPD due to the unpredictable disease trajectory.

We would like to emphasise that EoLC is an important part of every physician's daily work and an admission to hospital is a significant event in a patient's journey with chronic respiratory disease in terms of prognosis. SuISSA et al. [7] demonstrated that the $50 \%$ mortality following the first admission to hospital with an exacerbation of COPD was 3.6 years. Recognising and identifying patients entering the last year of their life can be particularly challenging in those with nonmalignant disease. It has been recognised that the division "active" and "palliative" care in respiratory medicine is not appropriate, and that the use of disease-modifying and symptom-relief-based strategies should run in parallel [8]. Compared with patients with lung cancer, those with COPD are much less likely to access palliative care services and more likely to be admitted to intensive care in the last 6 months of life [9]. Part of this may be down to different disease trajectories, but we must also recognise that there may be a failure of recognition and/or communication of the end-stage nature of the disease. The American Thoracic Society states that palliative care should be available near the end of life; however, they also emphasise that it should also be available at any point during the course of a progressive or chronic respiratory disease [10]. A crucial factor in achieving this is identification of patients, and communication of this information to the patients, carers and other health professionals involved in the care of the patient.

In conclusion, engagement of senior clinicians to act as champions for EoLC within their departments can improve palliative care for those patients though active communication with primary care. Consultations with these patients provide the opportunity to establish their priorities for care and begin the process of advance care planning as per their wishes. In doing so it has been shown that we can significantly improve the quality of life of our patients with end-stage respiratory disease.

- @ERSpublications

Improving end-of-life care for respiratory and COPD patients through better communication from secondary care http://ow.ly/U3ecw

Alison C. Boland ${ }^{1}$, Claire Hodgekiss ${ }^{1}$, Fiona Hicks ${ }^{2}$, Annette Edwards ${ }^{3}$ and Ian J. Clifton ${ }^{4}$

${ }^{1}$ Respiratory Registrar, Dept of Respiratory Medicine, St James's University Hospital, Leeds, UK. ${ }^{2}$ Consultant Palliative Medicine, Dept of Palliative Medicine, St James's University Hospital, Leeds, UK. ${ }^{3}$ Consultant Palliative Medicine, Wheatfields Hospice, Leeds, UK. ${ }^{4}$ Consultant Respiratory Physician, Dept of Respiratory Medicine, St James's University Hospital, Leeds, UK. 
Correspondence: Alison C. Boland, Respiratory Registrar, Dept of Respiratory Medicine, St James's University Hospital, Leeds LS9 7TF, UK. E-mail: alisonboland@nhs.net

Received: Sept 172015 | Accepted after revision: Oct 132015 | First published online: Dec 032015

Conflict of interest: None declared.

References

1 Diaz-Lobato S, Smyth D, Curtis JR. Improving palliative care for patients with COPD. Eur Respir J 2015; 46: 596-598.

2 Meffert C, Hatami I, Xander C, et al. Palliative care needs in COPD patients with or without cancer: an epidemiological study. Eur Respir J 2015; 46: 663-670.

3 Department of Health. More care, less pathway: a review of the Liverpool Care Pathway. 2013. www.gov.uk/ government/publications/review-of-liverpool-care-pathway-for-dying-patients Last accessed: August 24, 2015.

4 Department of Health. End of life care strategy: quality markers and measures for end of life care. 2009. www.gov. uk/government/publications/end-of-life-care-strategy-promoting-high-quality-care-for-adults-at-the-end-of-their-life Last accessed: August 24, 2015.

5 Guy's and St Thomas' NHS Foundation Trust. Amber care bundle. 2015. www.ambercarebundle.org/homepage. aspx Last accessed: June 17, 2015.

6 Leadership Alliance. One chance to get it right. Improving people's experience of care in the last few days and hours of life. 2014. www.gov.uk/government/publications/liverpool-care-pathway-review-response-to-recommendations Last accessed: August 24, 2015.

7 Suissa S, Dell'Aniello S, Ernst P. Long-term natural history of chronic obstructive pulmonary disease: severe exacerbations and mortality. Thorax 2012; 67: 957-963.

8 Bourke SJ, Peel ET. Palliative care of chronic progressive lung disease. Clin Med, 2014; 14: 79-82.

9 Au DH, Udris EM, Fihn SD, et al. Differences in health care utilization at the end of life among patients with chronic obstructive pulmonary disease and patients with lung cancer. Arch Intern Med 2006; 166: 326-331.

10 Lanken PN, Terry PB, Delisser HM, et al. An official American Thoracic Society clinical policy statement: palliative care for patients with respiratory diseases and critical illnesses. Am J Respir Crit Care Med 2008; 177: 912-927.

\section{QuantiFERON-TB performance enhanced by novel Mycobacterium tuberculosis- specific antigens}

To the Editor:

One of the most significant developments in the diagnosis of tuberculosis (TB) infection has been the introduction of whole-blood based interferon- $\gamma$ release assays (IGRAs) [1-3]. IGRAs, commercially available as QuantiFERON-TB Gold In-Tube test (QFT) (QIAGEN, Germantown, MD, USA) and T-SPOT.TB (Oxford Immunotec Ltd, Abingdon, UK), are based on the detection of a T-cell immune response towards RD1 antigens (ESAT-6 and CFP-10), with the addition of the TB7.7 antigen for the QFT only [1]. IGRAs are endowed with great specificity, as the antigens used are almost exclusively expressed by the Mycobacterium tuberculosis (MTB) complex, but not Mycobacterium bovis bacille Calmette-Guerin (BCG) [1-3]. However, the diagnostic sensitivity of IGRAs can be improved (75-85\% in HIV-negative active TB patients) especially in countries with a high TB burden [3].

To improve the performance of the IGRAs, addition of MTB antigens TB7.7, Rv3425 and EsxV to the current antigen cocktail has already been attempted $[4,5]$. Following a similar strategy, we evaluated the addition of six novel peptides to the QFT antigen cocktail to improve the diagnostic performance in terms of accuracy and dynamic range of interferon (IFN)- $\gamma$ level in active TB patients.

To this end, a multicentre study was performed under different clinical settings. Study group characteristics are reported in table 1 . Active TB patients $(n=85)$ were enrolled in three different clinical centres at the time of admission for being suspected of having TB and diagnosed according to Centers for Disease Control and Prevention/American Thoracic Society criteria [6]. Control subjects ( $\mathrm{n}=290)$ without any risk factors for latent TB infection were enrolled in two TB low-risk countries (Australia and Italy) 